\title{
Crohn's disease presenting with atypical mucocutaneous lesions in an 11 year old boy
}

\author{
Michael Sidiropoulos, Beth Skuy*
}

\section{CASE REPORT}

In April, an 11-year-old boy presented to our clinic with neck and anterior chest pain of 1 week's duration. Presenting symptoms included dysphagia and odynophagia. The patient's height and weight were in the 75th and 50th percentile respectively, and he had had stable growth since birth. His weight at presentation was $88 \mathrm{lbs}$. He was subsequently referred to a paediatrician who described a low-grade fever, occasional chills, decreased appetite, and difficulty swallowing both liquids and solids. At that time, while there was no cervical lymphadenopathy, he did have a few shallow ulcers on his lips. Within days, the patient developed periorbital edema, a small erythematous patch on the lateral surface of his left arm, and five, faint, mildly tender erythematous lesions on the fingers of his left hand. Examination of his oral cavity revealed extensive flat, erythematous vesicular-like lesions clustered on the midline of his lower lip, some of which had erupted to form ulcers. There were no other significant findings and the patient's past medical history was unremarkable. The patient was fully immunized and had no allergies. Laboratory investigations, including basic haematological and microbiological examinations, parasitology and urinalysis, yielded normal results. An x-ray of his neck and chest were normal, as was an upper GI series. An eye examination showed aseptic conjunctivitis.

The patient was subsequently evaluated by the department of Rheumatology at the local Children's Hospital and was diagnosed with "query Kawasaki disease," despite the insidious course of the illness. After receiving a course of intravenous

*To whom correspondence should be addressed: Dr Beth Skuy

The Scarborough Hospital, 462 Birchmount Road \#34

Scarborough, Ontario M1K 1N8 gamma-globulin, his symptoms quickly resolved and he was discharged home on low-dose Aspirin. He continued the Aspirin and was seen in the Pediatric Cardiology Clinic for follow-up 2 months later. Physical examination and an echocardiogram revealed no evidence of coronary involvement and the Aspirin was discontinued. The patient's weight had decreased to $85 \mathrm{lbs}$. Unfortunately, the patient complained of upper quadrant pain and fatigue on a daily basis throughout the summer and was seen by a paediatrician in October. His mother stated that his oral intake had decreased and he experienced occasional abdominal cramping following meals. Despite sleeping normally, the patient had decreased energy. There had been no fever, joint pain, rash or oral-ulcers. Bowel movements were

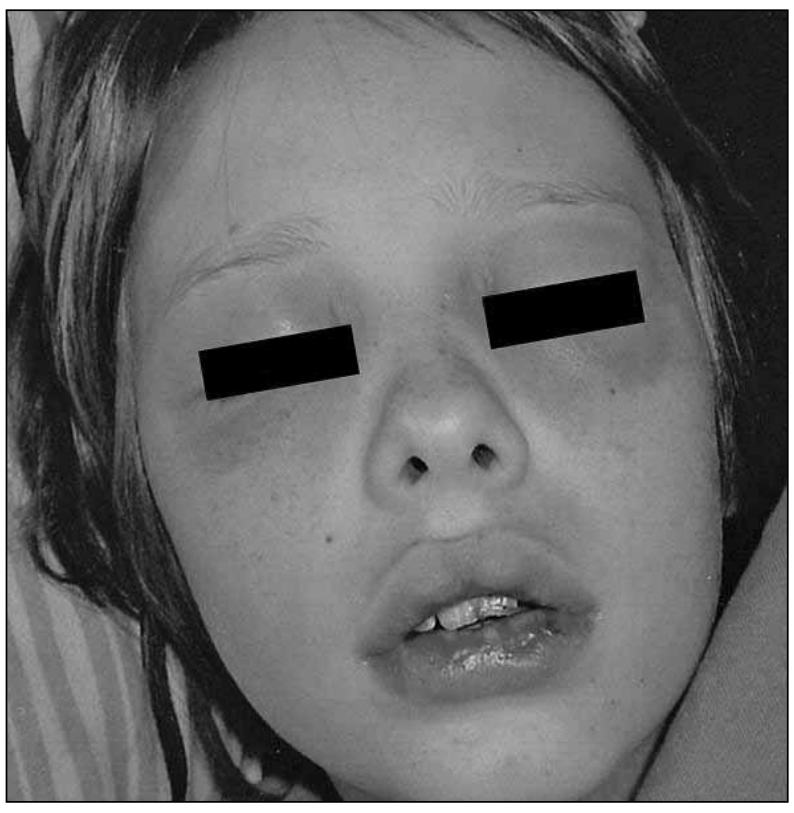

Figure 1. Extensive flat erythematous vesicular-like lesions are evident on the lower lip and periorbital erythema and edema is seen bilaterally. The patient complained of dysphagia and odynophagia. 
normal, with no blood in the stool and only occasional diarrhea. The patient was diagnosed with gastritis secondary to Aspirin use or cholelithiasis, since Kawasaki Disease can temporarily elevate lipids and triglycerides. A one month trial of Rabeprazole $20 \mathrm{mg}$ p.o. daily was prescribed. Blood work and an abdominal ultrasound were ordered and follow-up was scheduled within 1 month.

Two weeks following his presentation to the paediatrician's clinic, the patient presented to the local Children's Hospital with a sudden onset of severe right lower quadrant abdominal pain. He had a history of abdominal pain, anorexia and low grade fever for 3 days prior to presentation. An abdominal ultrasound revealed a perforated appendix with an abscess. He was admitted to hospital and put on Ampicillin, Gentamicin and Metronidazole. An abdominal CT scan showed a thickened appendix with an appendicolith present. There was also thickening of the terminal ileum and evidence of a fluid-filled abscess. Interventional Radiology was consulted and a drain was inserted to drain the abscess.

The Gastroenterology Service suggested that inflammatory bowel disease (IBD) might be a possibility, as additional information from the patient's mother revealed that her second cousin had Crohn's disease. Upon further investigation, the patient had an elevated erythrocyte sedimentation rate of $53 \mathrm{~mm} / \mathrm{hr}$ and a C-reactive protein of 26.9 $\mathrm{mg} / \mathrm{L}$. Albumin and total protein were both normal at $35 \mathrm{~g} / \mathrm{L}$ and $80 \mathrm{~g} / \mathrm{L}$ respectively. The patient weighted $81 \mathrm{lbs}$. A fistulogram was negative and the antibiotics were stopped after a total of 9 days. The patient returned to baseline and was scheduled for follow-up in the IBD Clinic within 2 weeks.

On presentation to the IBD clinic in November, the patient was still experiencing fatigue, poor appetite, and had some intermittently loose stools. There was no blood present in the stools. Physical examination was normal, except for mild clinodactyly. Diagnostic imaging revealed that the terminal ileum had mucosal irregularity in a "cobblestone" pattern. In addition, there was separation of adjacent bowel loops from the terminal ileum, with possible thickening of mesentery. A diagnosis of Crohn's disease was subsequently made. The patient was started on a course of prednisone which resulted in moderate relief of the gastrointestinal problems and stabilization of the patient's symptoms. Methotrexate or Immuran were subsequently added to the patient's regimen.

\section{DISCUSSION}

Crohns disease is a gastrointestinal disorder in which any part of the alimentary tract may become inflamed, from the mouth to the anus. The disease is characterized by relapsing and remitting signs and symptoms, the most common of which are abdominal pain, diarrhea, fever, and weight loss. However, there are numerous extraintestinal features, such as arthritis, iritis, and mucocutaneous lesions (1). Mucocutaneous lesions, although not considered part of the classic triad of fever, abdominal pain, and diarrhea, may be more common than previously thought. Mucocutaneous lesions have been reported to occur in $10 \%$ to $44 \%$ patients (2-5). Some of the more common lesions include erythema nodosum, pyoderma gangrenosum, Sweet syndrome, epidermolysis bullosa acquista and clubbing (6). Numerous oral lesions can also be present. These can include cobblestoning, mucosal tags, pyostomatitis vegetans and orofacial granulomatosis (7-9). Nonspecific lesions, such as aphthous ulcers, labial and facial edema and gingival erythema and edema are also common (9-11). These lesions are also known to precede gastrointestinal symptoms in patients with Crohn's disease $(19,12,13)$. Numerous studies have shown that oral lesions are often the initial presenting sign/symptom of IBD, particularly in children $(8-10,12,13)$. A study by Barnard and Walker-Smith found that $80 \%$ of pediatric patients with Crohn's disease had oral lesions (14). A study by Galbraith et al described 8 pediatric patients with asymptomatic Crohn's disease who presented with mucocutaneous lesions, of which the majority were oral and perianal lesions (9). The patient's ages ranged from 3 to 14 years old and common presenting lesions were lip swelling, oral ulcers or erosions, pyoderma gangrenosum and gingival edema. Many of these patients were also found to have perianal lesions, such as fissures, fistulas, and/or skin tags; however, in only 2 of the 8 patients was this one of the presenting complaints. The time to diagnosis of Crohn's disease after initial presentation with mucocutaneous lesions ranged from 3 months to 42 months. All patients within this study denied any gastrointestinal symptoms. We report a case of a pediatric patient who presented with mucocutaneous lesions, and was initially diagnosed with Kawasaki's, but was subsequently found to have Crohn's disease. Similar to previous reports, we suggest that Crohn's disease is a common cause of mucocutaneous lesions and that unexplained mucocutaneous lesions in a pediatric patient should alert the physician to further examine the patient to rule out Crohn's disease $(8,9,14)$. 


\section{REFERENCES}

1. Abraham C, Cho JH. Inflammatory bowel disease. N Engl J Med 2009;36:2066-76.

2. Peltz S, Vestey JP, Ferguson A, Hunter JA, McLaren K. Disseminated metastatic cutaneous Crohn's disease. Clin Exp Dermatol 1993;18:55-9.

3. Burgdorf W. Cutaneous manifestations of Crohn's disease. J Am Acad Dermatol 1981;5:689-95.

4. Marotta PJ, Reynolds RPE. Metastatic Crohn's disease. Am J Gastroenterol 1996;91:373-5.

5. Macaya A, Marcoval J, Bordas X, Moreno A, Vazquez S, Peyri J. Crohn's disease presenting as prepuce and scrotal edema. J Am Acad Dermatol 2003;49(2 Suppl Case Reports):S182-3.

6. Lebwohl M, Lebwohl O. Cutaneous manifestations of inflammatory bowel diseae. Inflamm Bowel Dis 1998;4:1428.

7. Berstein ML, McDonald JS. Oral lesions in Crohn's disease: report of two cases and update of the literature. Oral Surg Med Oral Pathol 1978;46:234-45.

8. Pittock S, Drumm B, Fleming P, McDermott M, Imrie C, Flin $\mathrm{S}$, Bourke B. The oral cavity in Crohn's disease. J Pediatr 2001;138:767-71.
9. Galbraith SS, Drolet BA, Kugathasan S, Paller AS, Esterly NB. Asymptomatic inflammatory bowel disease presenting with mucocutaneous findings. Pediatrics 2005;116:439-44.

10. Van de Scheur MR, Van der Waal RIF, Volker-Dieben HJ, Klinkenberg-Knol EC, Starink TM, Van der Waal I. Orofacial granulomatosis in a patient with Crohn's disease. J Am Acad Dermatol 2003;49:952-4.

11. Bogenrieder T, Rogler G, Vogt T, Landthaler M, Stolz W. Orofacial granulomatosis as the initial presentation of Crohn's disease in an adolescent. Dermatology 2003;206:273-78.

12. Ploysangam T, Heubi JE, Eisen D, Balistreri WF, Lucky AW. Cutaneous Crohn's disease in children. J Am Acad Dermatol 1997;36:697-704.

13. Plauth M, Jens H, Meyle J. Oral manifestations of Crohn's disease. An analysis of 79 cases. J Clin Gastroenterol 1991;13:29-37.

14. Barnard KM, Walker-Smith JA. The prevalence of oral manifestations of inflammatory bowel disease in a paediatric population [abstract]. J Dent Res 1994;73:A388.

Beth Skuy is a family doctor in scarborough ontario, and a teacher of both medical students and family practice residents.

Michael Sidiropoulos (Hon.B.Sc, M.Sc.,M.D.) is currently a third year resident in Anatomical Pathology at the University of Toronto. Michael obtained his Hon.B.Sc in Immunology, M.Sc in Cancer Epigenetics (Pathobiology and Laboratory Medicine), and M.D. all from the University of Toronto. He is also interested in completing a Ph.D in the near future, with a future career in academic medicine. 